\title{
Relationships with Pet Dogs and Well-being in Adulthood
}

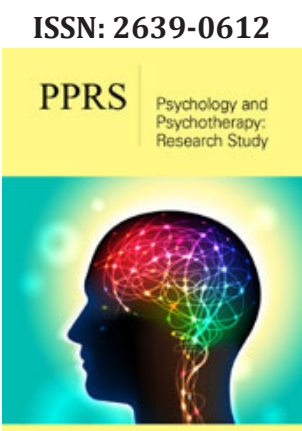

*Corresponding author: Effie Heotis, College of Arts and Sciences, USA

Submission: 柴 November 23, 2020

Published: 侮January 22, 2021

Volume 4 - Issue 4

How to cite this article: Effie Heotis. Relationships with Pet Dogs and Wellbeing in Adulthood. Psychol Psychother Res Stud. 4(4). PPRS. 000591. 2021. DOI: 10.31031/PPRS.2021.04.000591

Copyright@ Effie Heotis, This article is distributed under the terms of the Creative Commons Attribution 4.0 International License, which permits unrestricted use and redistribution provided that the original author and source are credited.

\author{
Effie Heotis* \\ College of Arts and Sciences, USA
}

\begin{abstract}
Despite that pets play an important role in contemporary society; little is known about the inner world of adults who have close relationships with dogs. The researcher conducted a qualitative, phenomenological study to develop a better understanding of the lived experiences of adults who have close relationships with dogs as companions. Attachment theory served to illuminate on the cognitive and developmental aspects regarding relationships with dogs during adulthood. Interviews were conducted with 15 adult dog owners to gain insights into the inner world of dog owners who have close relationships with dogs. Essential elements constituting the regulation of negative emotions, facilitation of positive emotions, promotion of increased physical activity, enhancement of interpersonal socialization emerged as core themes to shed light on adults' experiences of close relationships with dogs. Additionally, the companionship and security of unconditional love, and the fulfillment in meeting a loved one's needs emerged as central elements in adults' attachment experiences. This study provided an indepth understanding of how close relationships with dogs inform dog owners' psychological and physical well-being in adulthood. Implications of these findings are important for practices given that adults' wellbeing can be developed and promoted through close relationships with dogs.
\end{abstract}

Keywords: Well-being;Dogs;Pets;Attachment theory;Adult development;Counseling

\section{Introduction}

Companion animals, which are more commonly known as pets, are an important role in individuals' lives. Child psychologist Boris Levison was of the first to recognize the potential pets have in enhancing human health and well-being [1,2]. A landmark study conducted by Friedmann shed light the potential health benefits of pet ownership among heart patients. Despite that researchers for over two decades have provided scientific evidence that pets have the potential to promote many benefits for humans, little was known regarding the role of pets in human well-being was still lacking [3-5]. What continued to remain unresolved was the question of how pets can promote human wellness. Well-being is considered an important component of the human life span. However, little is understood as to how individuals' experience, achieve, and maintain well-being in their every-day lives [4,6]. Many scholars have suggested that pets play important roles in human health and well-being because they can be sources of comfort and companionship [3,7-13]. Given the potential implications of pets imparting well-being for their human companions, there was a dire need to developing an understanding of how pets influence human well-being. This study was conducted to contribute to a limited body of research on the benefits from adults' close relationships with their pet dogs. The development of an in-depth understanding of people's close relationships with their dogs can shed light on how pets provide opportunities to enhance mental, emotional and physical health via these social connections.

\section{Background}

In the 1960s, scientific researched evidence regarding the therapeutic effects of interactions between humans and animals emerged in the field of psychology. In the 1980s, scientists learned about the potential cardiovascular effects among heart attack patients who owned pets [1]. Despite these important findings, researchers only focused on therapeutic and physiological effects among young populations or populations that needed ongoing and specialized care [14-16]. Moreover, the main focus of the research pertained to the alleviation of negative signs and symptoms (i.e., depression, loneliness). As reflected in the lack of scientific research, an understanding of the lived experiences of normal, healthy adults' close relationships with their pet dogs remained scant. Attachment theory provided a lens to understand the emotional bond that develops in relationships. Attachment underlies healthy 
development throughout the human lifespan [13]. Attachment in adulthood affects one's ability to nurture ones' self as well as others.

\section{Data Collection}

Authorization was obtained from the institutional review board The researcher intended to obtain visceral views of participants as dog owners who have close relationships with their dogs and hear their voices. Given that experiences regarding relationships with pets can be quite subjective and nuanced [12], phenomenology was appropriate for this study. Rigor within a study was established to reflect on the trustworthiness, value, and meaningfulness of the study's findings. Measures undertaken to establish trustworthiness of the study included member checking, reviewing the newly developed instrument with an expert panel, field testing, reflexive journaling and maintaining a meticulously detailed audit trail. Fifteen interviews were conducted with adult participants via video conferencing applications and all interviews were audio recorded. The researcher also observed participants' situational and natural interactions with their dogs and developed field notes immediately after each interview. This interview protocol was best suited for this study given that participants were interviewed in their own homes whereby the relationships naturally develop and occur [9]. The data were analyzed using a thematic analysis procedure [17]. Six themes emerged in the study, which are discussed in the next section.

\section{Result}

Data analysis revealed that participants perceived their close relationship experiences with their dogs as promoting anxiolytic effects that contributed to their well-being. Participants indicted that the temperament of their pet dog enhanced their mental state and overall well-being, and especially during stressful situations. Participants' lived experiences of their close relationships with their dogs included assistance with regulation of negative emotions including stress, depression, grief, and loneliness. Providing care for dogs provided comfort, and close contact (e.g. cuddling and petting) was viewed as a source of comfort and companionship. Life enrichment and happiness was discussed at length by most participants. Participants reported that their lived experiences regarding their close relationships with their dogs provided a sense of joy and purpose, which they might not otherwise have experienced. Positive emotions also included expressions of feeling needed and loved. Participants were also sensitive and responsive to cues expressed by their dogs to indicating the need for feeding, grooming and exercise. Participants emphasized that they made it a priority to meet their dog's need for physical activity by sharing walks or runs together. Participants reported that their experiences regarding close relationships with their dogs included increased social interactions with neighbors and friends than they otherwise would have engaged in. Outings shared with the dog provided opportunities to start conversations with strangers met along the way.
Companionship and security of unconditional love also emerged as a prominent theme. This theme consistently emerged during discussions and it was expressed as a sense of feeling unconditionally loved, accepted, and comforted. Moreover, participants strongly expressed their attachment to their dogs as a source of unconditional love. Dogs were described as companions or family members that were non-judgement and always approving. Participants expressed that their attachment to their dogs is even more secure and fulfilling than attachments to humans could ever be. Central to attachment experiences was the sense of fulfillment in meeting a loved one's needs. Dogs were interpreted to communicate their needs and wants to their caretakers, and this was a prominent feature in attachment experiences. Providing care for dogs in turn generated a sense of fulfillment in meeting a loved one's needs and a positive outlook on life. A notable finding that emerged was that participants viewed their dogs as important members of the family and required the same quality of care compared to human members of family.

\section{Limitations, Summary and Conclusion}

This study pointed to the notion that dogs can be important for human health and well-being in adulthood. Phenomenology was used as a research paradigm to obtain a visceral view of participants. The research on the phenomena of relationships with dogs indicated that there are psychological and physical benefits to pet ownership. The present study provided insight on adult dog-owner relationships and embedded dynamics of attachment theory as the theoretical framework. Understanding how ownercompanion dog relationships promote positive well-being among adults may help develop key insights to inform professionals in mental health $[12,18,19]$. The information from this study may also be used as a basis for future therapies when treating dogowners with psychological challenges or who are experiencing stressful periods. Findings from this study can also provide practical information to help others make informed decisions on the potential benefits and challenges from owner-companion dog relationships in adulthood. Given that this study only explored the lived experiences of dog owners in the southeastern region of the US, future researchers could extend this study to include other geographical areas in the United States. Rental property owners could consider this study when ruling over the presence of pets in the rental property. Policies that enforce "no pets" on the premises are likely to force homeowners and condominium dwellers to relinquish their pets [20]. By simply excluding pet ownership in rental policies, the potential lessee forfeits the positive benefits of being a pet owner.

The implications of findings from this study also apply to policies pertaining to natural disaster planning. Findings from this study should be communicated to state and local emergency planners as they could consider this research when implementing policies and evacuation assistance programs for pet owners during 
emergency planning and natural disasters. Chadwin [21] found that during natural disasters such as hurricanes and floods, pet owners were unwilling to leave their pets behind due to lack of support and sufficient resources. Owners resisted evacuation orders and those who left their pets behind attempted to re-enter dangerous evacuation zones to rescue their pets $[21,22]$. Intense grief and depression were often experienced by individuals who were forced to abandon their pets during natural disasters [23]. Given these considerations, emergency planning protocols must be reformed to include the safety of pets.

Pets have been established as family members and ensuring human well-being and maintaining health. Pets have the potential to become catalysts to bring humanity together through crisis, while at the same time they require care and compassion [24-27]. Given that this study was not conducted during the Covid-19 Pandemic outbreak, future research could focus on the role of pets in managing or maintain well-being, as well as the concerns, stressors and difficulties in caring for pets during uncertainty and stress related to the Covid-19 pandemic. The dog owners who participated in this study expressed that pet dogs are reliable friends in good times and bad times. While providing companionship and unconditional love, dogs can contribute to the owners' well-being. It can be concluded that dogs can be "man's best friend". Relationships with dogs can be beneficial in many areas of an owners' life. Similar interspecies relationships and benefits accrued from them may be discovered in other parts of the world.

\section{References}

1. Anderson KA, Lord LK, Hill LN, McCune S (2015) Fostering the humananimal bond for older adults: Challenges and opportunities. Activities, Adaptation \& Aging 39(1): 32-42.

2. Vitztum C, Urbanik J (2016) Assessing the dog: A theoretical Analysis of the companion animal's actions in human-animal interactions. Society \& Animals 24(2): 172-185.

3. Hodgson K, Barton L, Darling M, Antao V, Kim FA, et al. (2015) Pets' impact on your patients' health: Leveraging benefits and mitigating risk. J Am Board Fam Med 28(4): 526-534.

4. Luhmann M, Kalitzki A (2018) How animals contribute to subjective well-being: A comprehensive model of protective and risk factors. The Journal of Positive Psychology 13(2): 200-214.

5. Martens P, Amiot C, Bastian B (2016) People and companion animals: It takes two to tango. BioScience 66(7): 552-560.

6. Hefferon K, Ashfield A, Waters L, Synard J (2017) Understanding optimal human functioning-The 'call for qual' in exploring human flourishing and well-being. The Journal of Positive Psychology 12(3): 211-219.

7. Baar M (2017) From working animals to cherished pets: Canine histories across the centuries. Groniek, Netherlands, p. 48.

8. Beck AM (2014) The biology of the human-animal bond. Animal Frontiers 4(3): 32-36.

9. Carter CS, Porges SW (2016) Neural mechanisms underlying humananimal interaction: An evolutionary perspective. In: Freund LS, McCune
S, Esposito L, Gee NR, McCardle P (Eds.), The social neuroscience of human-animal interaction. American Psychological, Washington, DC, USA, pp. 89-105.

10. Chandler CK, Fernando DM, Minton CB, Bethke PTL (2015) Eight domains of pet-owner wellness: Valuing the owner-pet relationship in the counseling process. Journal of Mental Health Counseling 37(3): 268282.

11. Heotis E (2017) Shaping social cognition though human-animal interactions. American International Journal of Research in Humanities. Arts and Social Sciences 19(1): 1-4.

12. Maharaj N, Kazanjian A, Haney CJ (2016) The human-canine bond: A sacred relationship. Journal of Spirituality in Mental Health 18(1): 7689.

13. Sable P (2016) The pet connection: An attachment perspective. Attachment 10(3): 199-210.

14. Hoagwood KE, Acri M, Morrissey M, Pierce PR (2017) Animal-assisted therapies for youth with or at risk for mental health problems: A systematic review. Appl Dev Sci 21(1): 1-13.

15. Jalongo MR (2015) An attachment perspective on the child-dog bond: Interdisciplinary and international research findings. Early Childhood Education Journal 43(5): 395-405.

16. McCune S, Esposito L, Griffin JA (2017) Introduction to a thematic series on animal assisted interventions in special populations. Applied Developmental Science 21(2): 136-138.

17. Braun V, Clarke V (2006) Using thematic analysis in psychology. Qualitative Research in Psychology 3(2): 77-101.

18. Amiot CE, Bastian B (2015) Toward a psychology of human animal relations. Psychol Bull 141(1): 6-47.

19. Hosey G, Melfi V (2014) Human-animal interactions, relationships and bonds: A review and analysis of the literature. International Journal of Comparative Psychology 27(1): 117-142.

20. Graham TM (2019) Enhancing access to quality rental housing for people with pets as healthy public policy (Calgary University).

21. Chadwin R (2017) Evacuation of pets during disasters: A public health intervention to increase resilience. Am J Public Health 107(9): 14131417.

22. Lowe SR, Joshi S, Pietrzak RH, Galea S, Cerda M (2015) Mental health and general wellness in the aftermath of Hurricane Ike. Soc Sci Med 124: 162-170.

23. Darroch J, Adamson C (2016) Companion animals and disasters: The role of human services organizations. Aotearoa New Zealand Social Work 28(4): 100-108.

24. Vincent A, Mamzer H, Ng Z, Farkas KJ (2020) People and their pets in the times of the COVID-19 pandemic. Society Register 4(3): 111-128.

25. Bowlby J (1969) Attachment and loss. Attachment. Basic Books, New York, NY, USA, Vol. 1.

26. Branson S, Boss L, Cron S, Kang DH (2016) Examining differences between homebound older adult pet owners and non-pet owners in depression, systemic inflammation, and executive function. Anthrozoös 29(2): 323-334.

27. Heotis E (2020) Phenomenological research methods: Extensions of Husserl and Heidegger. International Journal of School and Cognitive Psychology 7(2): 1-3. 